\title{
SELF-REPORTED PHYSICAL HEALTH AMONG THE AGED IN WUHAN, CHINA
}

\begin{abstract}
It has long been recognized that cross-cultural differences in health and illness reflect not only genuine variation in the incidence and prevalence of morbidity, disability, and mortality, but also the social processes by which these data were generated. These processes may be far removed from the biological reality. The conceptualization and measurement of physical health, therefore, can benefit, from adopting a comparative perspective. This paper aims to extend the generalizability of a three-dimensional model of self-reported physical health among the aged to China. Data came from a probability sample of approximately 2,700 urban and rural Chinese, aged 60 or older, living in the Wuhan area. The model, which consists of chronic illness, functional status, and self-rated health as three interrelated dimensions, was previously evaluated empirically using national data on the aged gathered in the U.S. and Japan. The model, which was found to apply equally well to the American and Japanese aged, also fit the Chinese data well. However, results also indicated cross-national differences (1) in the effects of age and sex on the prevalence of chronic disease and disability and (2) in the effects of education on disability and self-rated health.
\end{abstract}

Key Words: China, physical health, quality of life

This study aims to extend the generalizability of a three-dimensional model of physical health initially examined in two national samples drawn in the U.S. and Japan to a developing nation, China. Consisting of chronic illness, functional status, and self-rated health, this model was evaluated by analyzing data from a probability sample survey of approximately 2,700 older people in the Wuhan area, China. In addition, the effects of a set of selected sociodemographic variables on the multiple dimensions of physical health in the Chinese elderly were examined.

The assessment of physical health has played a prominent role in all phases of medical care, health administration, and planning. At the most general theoretical level, physical health is one of the three components of overall health as defined by the World Health Organization (1958). According to the WHO declaration, health is a state of complete physical, mental, and social well-being. However, if this broad definition of health is to guide health measurement and research, much effort needs to be directed to the explication and empirical evaluation of measurement models. These models provide an explicit characterization of not only the linkages between observable indicators and the latent variables but also interrelationships among the latent variables. These specifications may lead to valuable insights concerning the conceptual structure of selfreport physical health and the pattern of measurement errors involved.

Liang (1986) outlined a structural model as an initial step toward the explication of the multidimensional structure of physical health among the elderly. It specifies linkages among five dimensions of physical health. The chronic illness and disability days dimensions are medical definitions of health; the physical 
self-maintenance and instrumental activities of daily living dimensions are social definitions of health; and the subjective rating of one's own health is the psychological definition of health. The central theme of this model is that physical illnesses affect an individual's role performance, and subjective evaluation of one's health is a function of both illness and role performance. This model was empirically supported and the results were consistently replicated across four subsamples of the elderly drawn from the 1968 National Senior Citizens' Survey conducted in the United States.

A simplified three-dimensional model was recently suggested and examined (Whitelaw and Liang 1991; Liang, Bennett, Whitelaw, and Maeda 1991). The three dimensions were labelled chronic illness, disability, and self-rated health. Composites instead of multiple indicators were used to measure the chronic illness and functional status dimensions. This three-dimensional specification of physical health was empirically evaluated and supported by data from national samples of the aged in the United States and Japan.

It has long been recognized that cross-cultural differences in health and illness reflect not only genuine variation in the incidence and prevalence of morbidity, disability, and mortality, but also the social processes by which these data were generated. These processes may be far removed from the biological reality. The conceptualization and measurement of physical health, therefore, can benefit, from adopting a comparative perspective. A major assumption underlying this approach is that beliefs about health, diseases, and medical treatment are the product of a particular historical, social, and cultural setting (Lock 1987; Ikels 1991).

Empirical studies concerning the properties of health measures have largely been based on data collected in the developed nations, particularly the United States. Observations from non-western developing societies are especially useful in assessing the construct validity as well as generalizability of the existing findings regarding the structure of physical health. As suggested by Kohn (1987), in no other way can one be certain that observations from Western cultures are not merely the product of some limited set of historical, cultural, or political circumstances.

Given the substantial differences between its culture and those of the United States and Japan, China provides an ideal setting to evaluate the construct validity and external validity of the three-dimensional model of physical health. Because the physical health status measures were initially developed in the U.S., it is reasonable to suspect that not all the items are equally useful in China. A given item which was valid and reliable in the U.S. may be unacceptable when administered in China (Ikels 1991). The Chinese data are relevant to the assessment of construct and external validity of self-reported health status because they differ significantly from the data collected in the United States in terms of cultural context. In the present study, these data were used not for the purpose of comparative analysis in that cultural differences are hypothesized to be the major variables accounting for the observed differences. Instead, the Chinese data were utilized largely because they provide an opportunity for 
replications at a different time, in a different setting, with a body of data quite different from those derived from the United States.

To place the present study in a meaningful context, a brief overview of the demographic and health trends as well as current research on health status in China is in order. As a developing nation, China had more than 1.1 billion people in 1990, accounting for more than 20\% of the world's population (State Statistical Bureau of the People's Republic of China 1991). Although persons aged 65 and over constituted only $5.58 \%$ of its total population in 1990, China also has the largest population of elderly people in the world, numbered more than 63 million.

China's population structure has undergone some significant changes in recent decades. The total fertility rate declined from some 6 births per woman before 1970 to 2.5 in 1982. Life expectancy at birth was about 35 years before 1949, and it increased to 67.9 years in 1981 (Coale 1984; Jaing, Zhang, and Zhu 1984). As a result of demographic transition, in particular a declining fertility, China will have a substantially older population in the middle of the 21 st century. It is projected that by 2050 , the percentage of the population aged 65 and over may be as high as 13 to $18 \%$ (Banister 1988; Liang, Tu, and Chen 1986; Grigsby and Olshansky 1989). In comparison with the western developed nations, the aging of the Chinese population is going to be much more accelerated. For example, while in France, Sweden, United States, and United Kingdom, it took from 45 to 130 years for the 65 -plus population to grow from 7 to $14 \%$, in China this process is projected to take only 25 years (International Assistance Group on Family Planning, Japan 1989).

Parallel to the processes of demographic transition and population aging is the epidemiologic transition in China. Before 1949 periodic epidemics and a high level of infectious disease morbidity and malnutrition existed in China, hence its reputation as the "sick man of East Asia". During the past four decades, substantial improvement in population health has resulted not only in increased life expectancy but also the emergence of a new pattern of causes of morbidity and mortality. In urban areas and much of rural China, parasitic and infectious diseases have been substantially replaced as causes of death by stroke, cancer, heart disease, accident, and chronic respiratory diseases (Jamison, Evans, King, Porter, Prescott and Prost 1984; Kantha 1990; Xu 1985).

However, there are still considerable disparities in population health and health care between urban and rural areas in China. Although the epidemiologic transition is almost completed in the urban areas, such a process is still progressing in the rural areas. Some communicable diseases such as dysentery, tuberculosis, and hepatitis remain important national problems. In poor regions of China, health conditions may lag 20 years behind those attained in more developed areas. Whereas in urban areas malnutrition has been substantially reduced and is no longer a major problem, many children in rural areas continue to suffer from malnutrition. In addition, there are substantial urban-rural differentials in health care. In the early 1980 s, more than $30 \%$ of the Chinese population were completely uninsured, virtually all of them rural residents. 
Furthermore, health care expenditure in urban areas is more than triple that estimated for rural expenditure per capita, and state subsidies for health care for urban dwellers are at almost ten times those for rural dwellers (Jamison et al. 1984). Since the early 1980 s the medical care system has suffered from a lack of investment because it is not a 'production sector'. In the countryside where some $80 \%$ of the Chinese reside, the cooperative health care system has largely disintegrated, and most must pay.

Broadly, there are at least three approaches toward the analysis of health status among the Chinese elderly. The first approach involves a macro-social, demographic, and epidemiologic analysis of mortality and morbidity of the total population in China (Jamison et al. 1984; Kantha 1990; Xu 1985; Yang, Lin, and Lawson 1991). Although these studies are informative with reference to societal, demographic, and health trends in China, their foci are primarily on the total population as a whole instead of the older population. Population health is assessed largely in terms of highly aggregated and often indirect measures of mortality, morbidity, and nutritional status. Micro-level data pertaining to health related quality of life in the aged such as disability and health perceptions are generally not available.

The second approach entails qualitative and/or small-scale surveys (Ikels 1991; Yang 1989). Whereas some of these studies contain a rich depiction of the sociocultural context of health status of the aged in China, the analyses are generally descriptive and frequently without an explicit and well articulated conceptual framework. For example, dimensions of health status such as diseases, disability, and self-rated health exchanges are often not explicated, and their interrelationships unexplored. Given that the samples used are rather small and most likely from urban areas, it is difficult to generalize the findings from these studies.

The third approach includes large-scale sample surveys of the Chinese elderly (e.g., Gu and Wang 1989; Gui, Ki, Shen, Di, Gu, Chen, and Qian 1987; Population Research Institute, CASS 1988; Qu 1984). Although great efforts were devoted to generate statistics representing a broad range of the Chinese aged, these studies in general did not employ a probability sampling design. Their survey questionnaires are often limited in terms of the measures of health related quality of life, and the design and execution of the research operations are of uneven quality. As suggested by Kantha (1990), Chinese health statistics are often spotty and too politicized to draw any hard and fast conclusions. In addition, statistics are difficult to verify and frequently presented in a noncomparable format. More importantly, the analyses rarely go beyond descriptive analysis. Multivariate analysis involving causal modeling is rare.

The present research aims to contribute to the current knowledge on health status of the Chinese aged in the two following ways. First, on the basis of data collected from a large-scale probability sample survey of some 2,700 elderly people in the Wuhan area in China, descriptive statistics of the socioeconomic conditions and physical health will be presented. Second, an explicit model depicting the multi-dimensional structure of physical health status and its relations with socioeconomic variables will be evaluated. 


\section{MODEL SPECIFICATIONS}

\section{Measurement Model}

Figure 1 presents a diagram of the three-dimensional model of self-reported physical health which is in turn assumed as a function of socioeconomic variables $\left(\mathbf{M}_{1}\right)$. Here, $\xi$ 's (xi's) and $\eta$ 's (eta's) are the latent variables, and the $\zeta$ 's (zeta's) represent residual errors in the structural equations. The $\beta$ 's (beta's) are regression coefficients associated with the latent variables. The x's and y's signify the observable indicators. All variables, latent and observed, are measured in deviations from their means. The $\lambda$ 's (lambda's) are the factor loadings or the regression coefficients of the observed indicators on the latent variables. The arrows signifying the effects of the exogenous variables on the physical health dimensions are denoted as $\gamma$ 's (gamma's). Finally, the $\delta$ 's (delta's) and $\varepsilon$ 's (epsilon's) denote measurement errors associated with the observable indicators. To simplify presentation, correlations among the $\xi$ 's are not depicted. The notations used here are in accordance with the LISREL conventions suggested by Jöreskog and Sörbom (1988).

According to Figure 1, self-reported physical health consists of three dimensions: physical illness $\left(\eta_{1}\right)$, functional status $\left(\eta_{2}\right)$, and self-rated health $\left(\eta_{3}\right)$. These dimensions reflect three different approaches in measuring physical health. As the medical definition of health, physical (chronic) illness $\left(\eta_{1}\right)$ is defined by the presence of disease or impairment. Functional status $\left(\eta_{2}\right)$, the social definition of physical health, is defined by the ability to function in normal social roles and social settings. As a psychological definition, self-rated health refers to the individual's perception and evaluation of his or her physical health. Self-rated health $\left(\eta_{3}\right)$ is hypothesized to be a function of physical illness $\left(\eta_{1}\right)$ and functional status $\left(\eta_{2}\right)$, whereas functional status is assumed to be a consequence of physical illness.

As shown in Figure 1, chronic illness, functional status, and self-rated health are measured respectively by 1,3 , and 4 indicators. Only independent random measurement errors in these indicators are assumed. Additional detail concerning the specific indicators will be provided in the measurement section.

\section{Relationships with Exogenous Variables}

Although self-reported health is hypothesized to contain three interrelated dimensions, they are nevertheless distinct. This distinctness can be further illuminated by studying the impact of the exogenous variables such as age $\left(\xi_{1}\right)$, sex $\left(\xi_{2}\right)$, education $\left(\xi_{3}\right)$, marital status $\left(\xi_{4}\right)$, and urban/rural residence $\left(\xi_{5}\right)$ on the three physical health dimensions. Since there have been few multivariate analyses of the physical health status of the Chinese elderly, the specifications were largely proposed on the basis of prior research in the United States. In particular, we hypothesize that the old old (i.e., 75 or older) and women tend to experience more chronic illness and functional impairments (Fillenbaum 1979; 


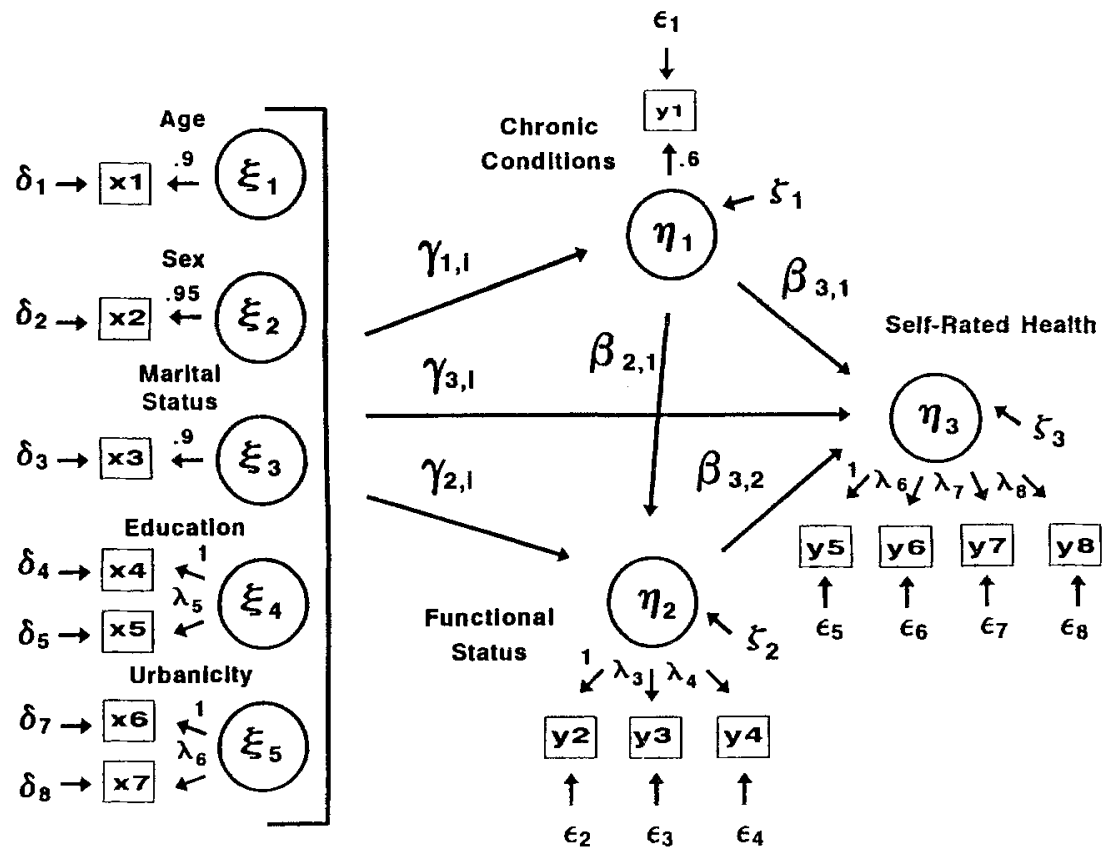

Fig. 1. Model of self-reported physical health $\left(\mathrm{M}_{\mathrm{l}}\right)$.

Markides and Martin 1979; Stoller 1984). In addition, the old old and women tend to rate their own physical health as better than the young old (i.e., 65 to 74 years of age) and men (Ferraro 1988; Fillenbaum 1979; Stoller 1984). Such age and sex differences in self-rated health persist even when objective health variables are controlled. Although the impact of education and marital status of the older person on chronic illness and functional impairment is not conclusive, there are some observations that higher education and being married may lead to better objective health and subjective health (Angel and Thoits 1987; Lock 1987). In view of the substantial urban/rural differences in socioeconomic development, population health, and health care (Jamison et al. 1984; Liang, Tu, and Chen 1986), urban residents are hypothesized to be better off in terms of chronic diseases, functional status, and self-rated health.

With reference to the interrelationships among age, sex, education, marital status, and urban/rural residence, the following correlations are postulated. First, age is correlated with the female gender, lower education, and being widowed. Second, older women are hypothesized to have relatively less education and are more likely to be widowed. Third, a positive correlation is assumed between being married and education (U.S. Senate Special Committee on Aging 1988). Fourth, urban residence is hypothesized to be associated with higher average education but is uncorrelated with age and sex composition. 
METHODS

\section{Setting}

China is a vast and heterogeneous county. There are substantial regional differences in socioeconomic development and sharp urban-rural inequalities. Due to many political and practical constraints, at the present time it is extremely difficult if not impossible to undertake a national survey of the aged in the Chinese mainland. A sensible initial strategy is to conduct research in one or more well defined locations typical of the social and cultural conditions in China. As one's experience accumulates, expansion can be made to realize eventually the goal of a national survey of the Chinese elderly. The basic design of the present research illustrates such an approach.

Data for this research came from the 1991 Survey of Health and Living Conditions of the Aged in the Wuhan Area in China. The survey, designed by the investigators at the University of Michigan and Wuhan University in conjunction with the staff of the Wuhan Bureau of Statistics, involved a threestage probability sample of the elderly population aged 60 and over residing in the Wuhan area on July 1, 1990, based on the 1990 Census in China (Liang, Gu, and Krause 1992).

Wuhan is located in the province of Hubei in south central China. Geographically, Hubei is a part of the middle and lower Yangtze River Basin, which is China's most important waterway. Oceangoing vessels can navigate up to Wuhan which, along with Shanghai, is a major industrial city. Major industries include steel making, machine manufacturing, and textiles. With hot, humid summers and a 9 month growing period, the Yangtze Basin is China's most productive agricultural region. The major crops include grain, cotton and vegetables.

The Wuhan area is very typical of the Chinese mainland in terms of social and economic conditions and geography. As part of the general policy to promote social and economic development in the rural areas, many Chinese municipalities have been extended to include the rural regions nearby. Administratively, Wuhan consists of 7 urban districts, 2 suburban districts including state farms, and 4 rural counties. Therefore, Wuhan not only contains the city proper and suburban areas, but also encompasses substantial rural areas including mountain villages, lake towns, plain areas, and state farms.

People 60 years of age or older in the Wuhan area constituted about 8 percent $(622,574$ persons) of the total population of 6.9 million in 1990 . With an urban and suburban population of approximately 4.04 million, Wuhan is the fifth largest city in China. The four rural counties cover an area of $6,840 \mathrm{~km}^{2}$, and contained a total population of 2.86 million in 1990 . Between 1982 and 1990 , the urban and suburban areas experienced substantially higher population growth (from 11 to $35 \%$ ) than the rural areas (6 to 13\%). In the urban and suburban areas, some 26 to $53 \%$ of the population was engaged in industrial production in 1990 , but only 7 to $17 \%$ of the rural population was so engaged. 
As expected, the urban areas are much better off socioeconomically. Per capital income was 1270 yuan $(\$ 245)$ in the urban areas and 645 yuan $(\$ 129)$ in the rural areas. In 1988, life expectancy at birth was 71.4 years. The illiteracy rates in the urban districts ranged from 7 to 11 percent, substantially lower than those in the suburban districts and rural counties (18 to $28.5 \%$ ).

\section{Data}

Questionnaire development for this study involved the translation of numerous scales and items from English into Chinese and their modifications in response to the unique setting in the Wuhan area. Each item was intensively examined to ensure the quality of translation. Several portions of the questionnaire had previously been field tested (Gu and Guo 1989; Gu and Wang 1989). However, the entire questionnaire was nevertheless subjected to several critical evaluations including one focus group discussion, two pretests, and a pilot study involving 50 elderly persons.

Out of 3,551 names selected from the 1990 Chinese Census records, interviews were successfully completed with 2,938 individuals including 169 proxy interviews. Proxy interviews were conducted when the designated respondents, because of physical and/or mental impairments or any other reasons, could not be interviewed directly. This resulted a response rate of $83 \%$. Major reasons for the 613 non-responses included (1) death (34\%), (2) inability to locate the selected respondent at the given address (31\%), (3) temporary absence (18\%), and (4) other reasons (13\%). Only six individuals refused to be interviewed, and four did not participate because of health reasons. If the deceased were excluded as ineligible to participate in the study, the response rate would increase to $88 \%$. In addition to the cases with incomplete data $(n=19)$, proxy interviews were excluded in the current study due to the self-reported nature of the items in the analyses, thus reducing the effective sample size to 2,755 .

Given the non-respondents described above and the exclusion of proxy interviews, to what extent were the most impaired excluded from our analyses? Due to a very high response rate, the likelihood of excluding the most impaired from the survey is relatively small. However, as one would expect, the proxy respondents are substantially more impaired than the self-respondents in terms of selected chronic diseases (i.e., hypertension, diabetes, stroke) and functional status (i.e., ADL, IADL, and fitness). Nevertheless, the issue is to what extent the effective sample represents the total sample adequately. With reference to descriptive statistics, the effective sample does understate the prevalence of disability and certain diseases by 1 or 2 percent. With regards to the results of multivariate analyses, the effect of such underestimations is unknown. Current knowledge regarding the effect of survey and item non-response is very limited. Although imputation of missing data is possible, its merits are highly debatable. 


\section{Measurement}

Socioeconomic characteristics. Age was measured in chronological years. Sex and marital status are coded as dummy variables such that 1 represents being female for sex, and being currently married for marital status. Education was assessed by two measures: (1) formal schooling in years, and (2) the level of education as a result of formal and informal schooling including traditional tutoring and remedial adult education. Finally, urbanicity was indexed by two dummy variables: (1) urban/rural district and (2) non-agricultural/agricultural household, with urban and non-agricultural coded as 1. Because age, sex, and marital status were measured by single-indicator measures, factor loadings and measurement error coefficients were fixed according to known reliabilities $(0.90,0.95$, and 0.60 , respectively) derived from the 1970 U.S. Census (1975). Given that the authors have no firm information concerning these reliabilities in China, it seemed more reasonable to use estimates from the U.S. than to assume no error at all.

Health characteristics. Chronic conditions reflects a simple count of "yes" responses to two lists of 23 items assessing the presence of a condition or disease. Reliabilities of self-reported chronic conditions are assumed to be 0.6 on the basis of previous research (Elinson 1988). Functional status was measured by three indicators, activities of daily living (ADL), instrumental activities of daily living (IADL), and physical fitness, that reflected composites of 6,6 , and 10 items, respectively. These composites were coded such that a higher score reflects more difficulties. Finally, self-rated health was comprised of 4 items, with all items coded such that a higher score reflects poorer health. These items included: "present health status" ("excellent", "very good", "good", "fair", and "poor"), "health compared to others your own age" ("better', "same', or 'worse'), and satisfaction with health ("completely satisfied", "very satisfied", "somewhat satisfied", "not very satisfied", and "not at all satisfied").

\section{Data Analysis}

A descriptive analysis of socioeconomic characteristics and physical health was conducted first. Then, on the basis of the proposed framework (see Figure 1), the interrelationships among socioeconomic variables and the dimensions of physical health were analyzed using LISREL 7, a widely used statistical program for estimating structural equation models (Jöreskog and Sörbom 1988). Structural equation models seek to explain the relationships among a set of observed indicators in terms of the latent variables or theoretical dimensions. Relationships among the indicators are characterized by their covariances and it is assumed that the latent variables are generating the relationships among the indicators that are revealed in the covariance matrix (Bollen, 1989). In addition, data analyses were replicated to minimize the capitalization of chance. Specifi- 
cally, the sample was randomly divided into two subsamples. One subsample was analyzed first for exploratory purposes to test the model for acceptable fit. The analysis was then replicated by using the other subsample. Convergent findings from multiple replications further reinforce one's confidence in the results. After excluding cases with missing data, the effective sample size for the total sample was 2,755 . The random split resulted in sample sizes of 1,378 and 1,377 for subsamples 1 and 2 , respectively.

\section{RESULTS}

\section{Descriptive Analysis}

Socioeconomic background. Table I presents the frequency distributions of socioeconomic characteristics. In comparison with the elderly populations in the developed nations, the sample exhibited a relatively young age composition. Some $60 \%$ of them were between 60 and 69 years of age, $34 \%$ were in their 70 's, and only $6 \%$ were in the 80 s. Consistent with the observed sex difference in mortality, $55 \%$ of the respondents were women. A greater percentage of those in their 70 s or older (i.e., the older old), were women. About $62 \%$ of the sample were married, while $37 \%$ were widowed. The older old and women were more likely to be not married.

Due to the nature of the sample, $59 \%$ of the respondents resided in the urban area. This level of urbanization is much higher than that $(26 \%)$ for China as a whole (State Statistical Bureau of the People's Republic of China 1991). The older old, women, and those not married were more likely to be residing in the rural areas.

The Chinese aged generally have very little education. Close to $60 \%$ of the respondents were illiterate, whereas only $22 \%$ of the population aged 15 and over were illiterate in 1990 (State Statistical Bureau of People's Republic of China 1991). The rates of illiteracy were particularly high among those 70 years of age or older $(68 \%)$, women $(81 \%)$, those not married $(74 \%)$, and rural residents $(72 \%)$.

Health characteristics. The percentage distribution of health characteristics by age, sex, marital status, and residence are presented in Table II. First, diseases and conditions are described item by item, and as a composite. Next, the items included in the three composites representing the functional limitations dimensions are examined. This includes six ADL, six IADL, and ten physical fitness items. Finally, the four self-rated health status items are described.

The ten most reported conditions or diseases among the Chinese elderly in the Wuhan area were arthritis or rheumatism (47\%), chronic back pain (44\%), respiratory disease $(30 \%)$, anemia $(26 \%)$, high blood pressure $(23 \%)$, heart disease $(20 \%)$, stomach or intestinal ulcers $(20 \%)$, cataracts $(19 \%)$, bedsores or 


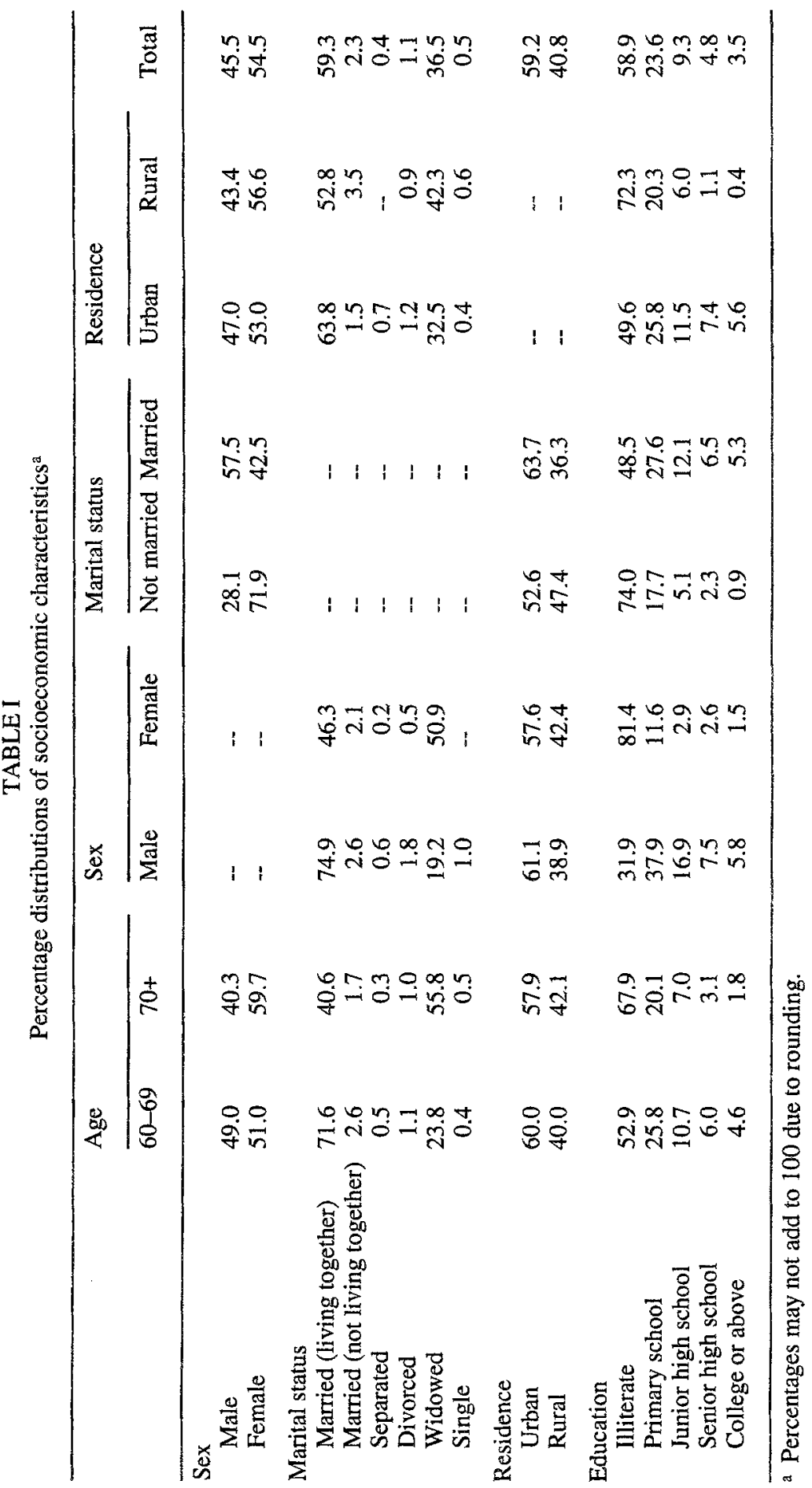




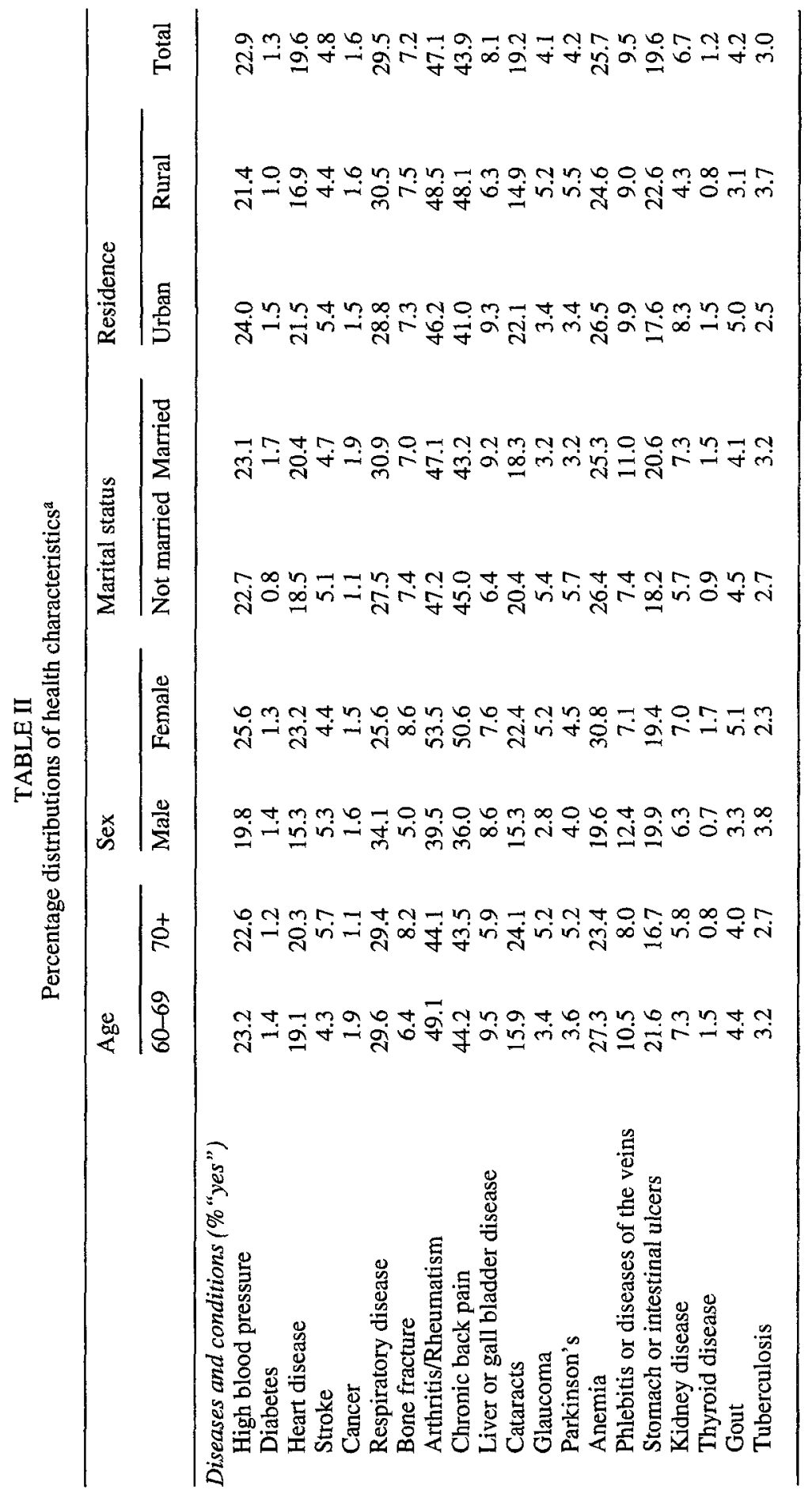




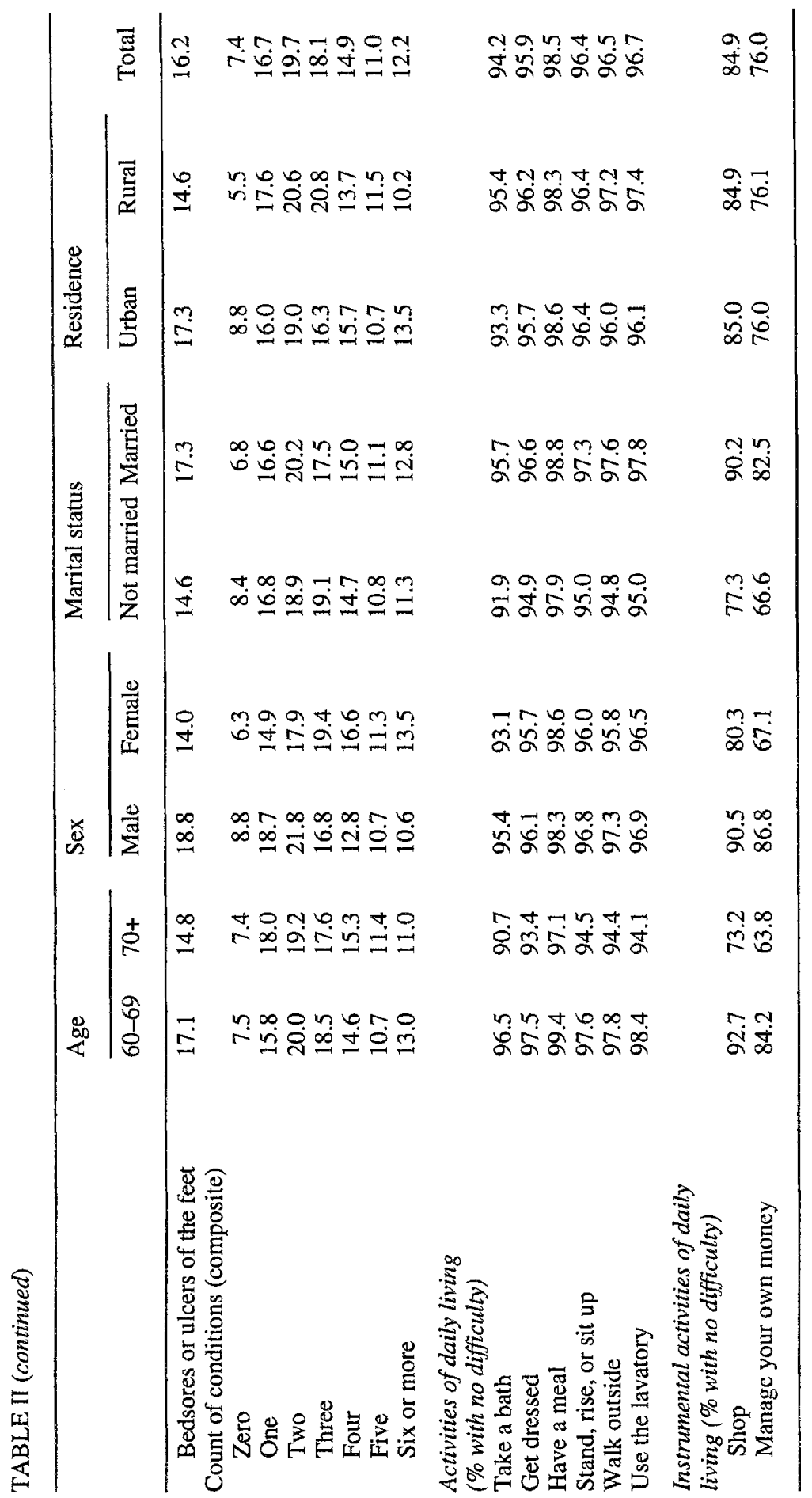




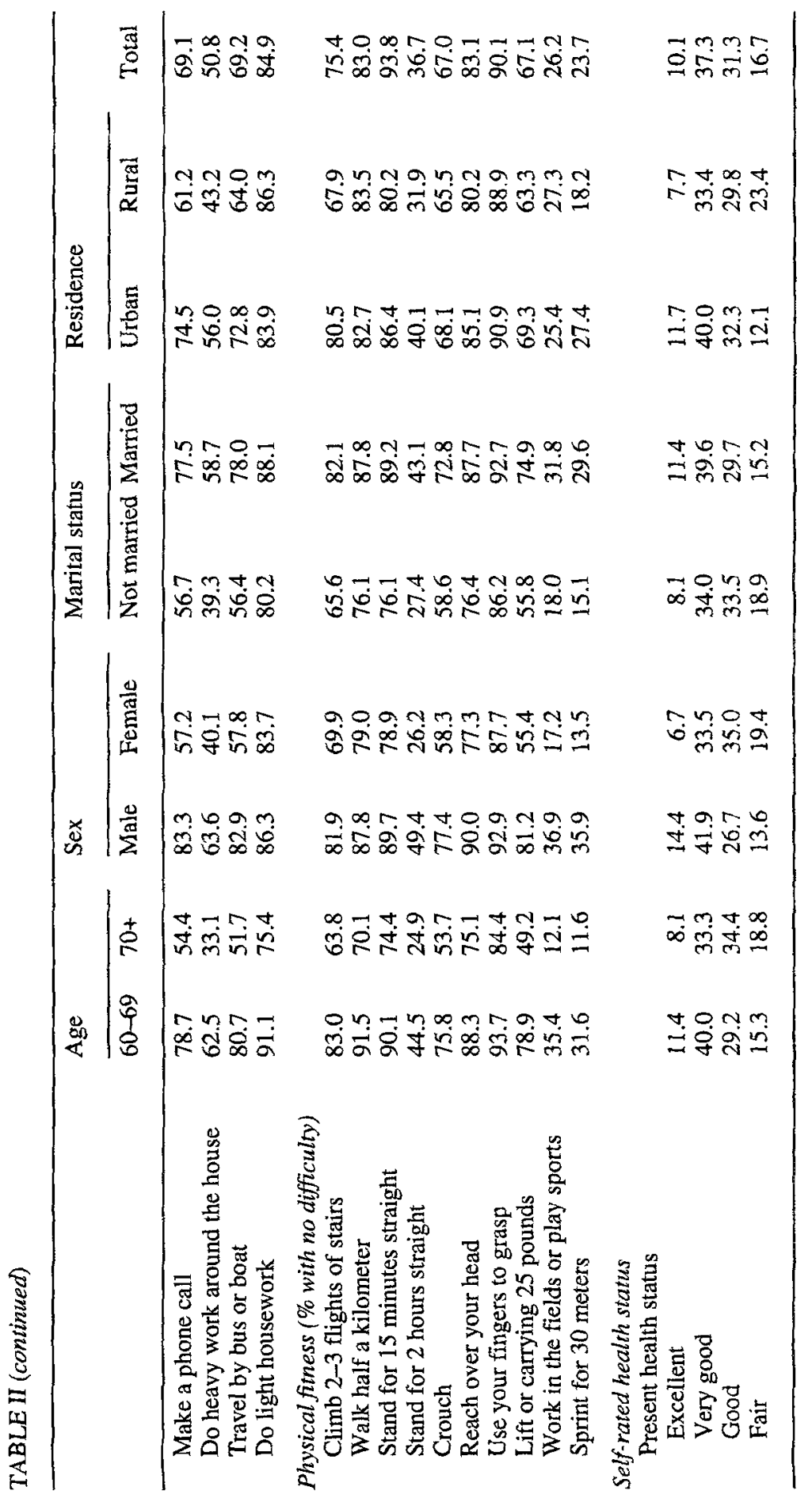




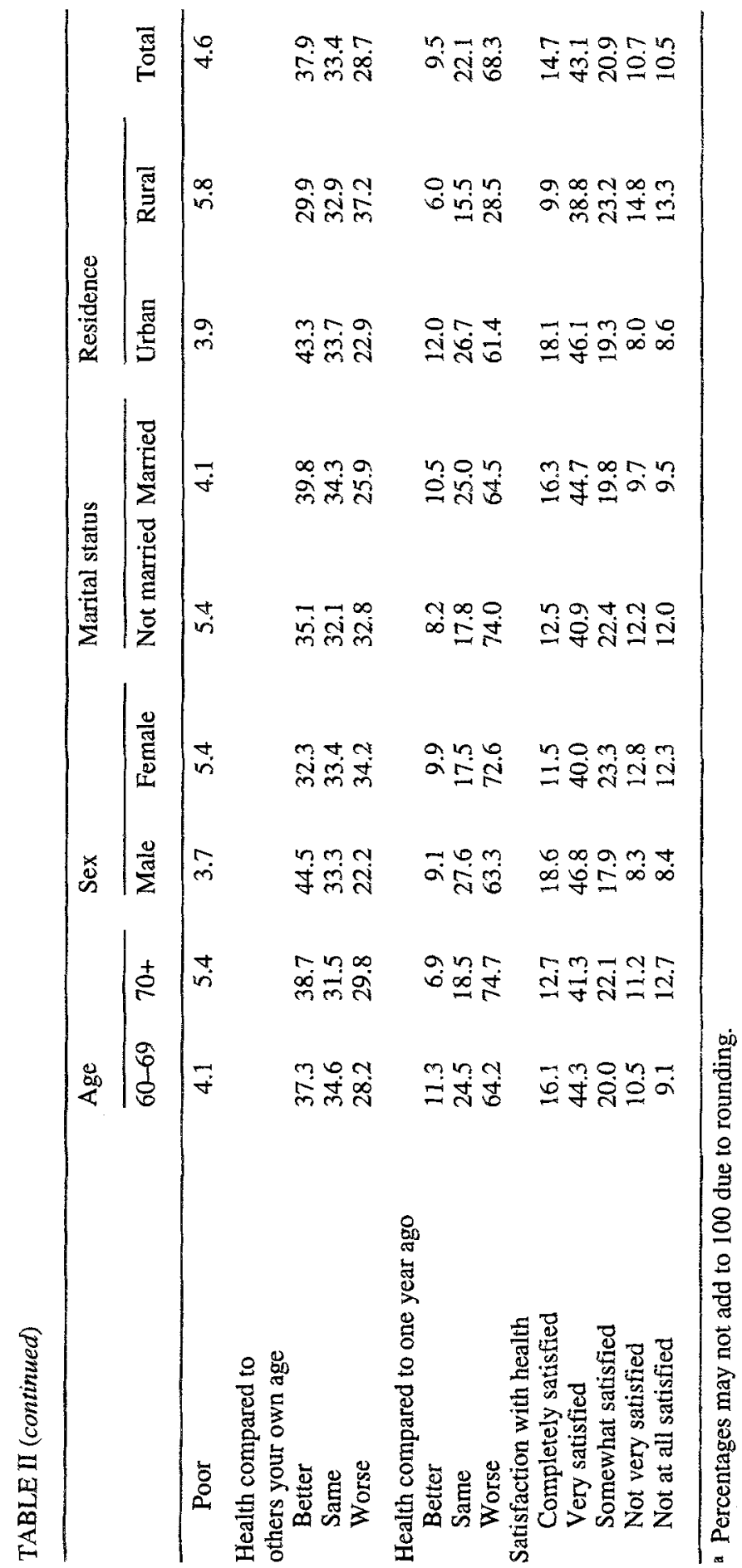


ulcers of the feet (16\%), and phlebitis or diseases of the veins (10\%) (Table II).

There appeared to be a good deal of sociodemographic variations in selfreported prevalence of diseases. In particular, a slightly greater percentage of individuals 60 to 69 years of age had arthritis/rheumatism, anemia, liver or gall bladder disease, phlebitis, and stomach or intestinal ulcers. However, stroke, fracture, cataracts were more prevalent among the older old.

When examining sex differences, women appeared to have a greater percentage of reported cases of arthritis/rheumatism, chronic back pain, cataracts, glaucoma, anemia, high blood pressure, heart disease, and bone fractures. Males seemed to have a higher percentage of cases of respiratory disease, phlebitis or diseases of the veins, tuberculosis, and bedsores or ulcers of the feet.

Regarding marital status, the percentage of married elderly who reported having diabetes and thyroid disease was almost twice that of those not married. In addition, there was a slightly higher percentage of married aged who reported respiratory disease, liver/gall bladder disease, phlebitis, and stomach conditions. On the other hand, not married elderly evidenced a higher percent of individuals with cataracts, glaucoma, and Parkinson's disease.

Diseases more prevalent among the urban residents included high blood pressure, heart disease, liver or gall bladder disease, cataracts, anemia, and kidney disease, whereas rural residents were more likely to suffer from chronic back pain, stomach or intestinal ulcers, and slightly higher percentages of glaucoma and Parkinson's.

Taking all conditions together, only $7 \%$ of the respondents did not report any disease, $36 \%$ reported one or two conditions, and $56 \%$ had three or more conditions. The older old, women, and those not married appeared to suffer from more chronic conditions, whereas there was no major urban/rural difference.

The elderly in the Wuhan area evidenced only a small amount of ADL limitations. The percentage with no ADL difficulty ranged from $94 \%$ (bathe) to 99\% (have a meal). Looking at the ADL items by age, it is not surprising that for all items, a slightly smaller percentage of those in the 70 and over age group were able to do the activities by themselves with no difficulties. In addition, there did not appear to be any major difference by sex, marital status, or residence.

There was a much wider range in the percentage of elderly able to perform IADL tasks by themselves with no difficulty. While $85 \%$ of the respondents were able to shop and do light housework by themselves with no difficulty, only $76 \%$ were able to manage their own money, $70 \%$ to make a phone call or travel by bus or boat, and only $51 \%$ were able to do heavy housework.

In all cases, younger Chinese elderly, males, and those currently married were more likely to perform the IADL's by themselves with no difficulty. Rural elderly seemed to have more difficulty making a phone call, doing heavy work around the house, and traveling by bus or boat. These differences might be due to the limited exposure that rural elderly have to modern conveniences.

In addition to ADL and IADL, functional status can also be measured in terms of physical fitness or limitation which is an expression of a person's capability 
instead of the demand of the environment (Pope and Tarlov 1991). Concerning physical fitness, not surprisingly only 24,26 , and $37 \%$ of the respondents were able to sprint for 30 meters, work in the fields or play sports, and stand for two hours with no difficulty by themselves. However, the remainder of the items indicate a fairly fit group of individuals with a range between 67 and $94 \%$ able to perform the activities by themselves with no difficulty.

Again, Chinese aged 60 to 69 , males, and those currently married showed a greater percentage of individuals able to perform the activities by themselves with no difficulty. Urban elderly reported a higher percentage able to climb two to three flights of stairs, stand for 15 minutes, stand for two hours, reach over their head, lift/carry 25 pounds, and sprinting for 30 meters.

With reference to self-rated health, a large majority (79\%) of the Chinese respondents considered themselves in good to excellent health, whereas only $21 \%$ reported their present health as fair or poor. Compared to others their own age, $38 \%$ of the Chinese elderly felt their health was better, $33 \%$ the same, and $29 \%$ worse. In addition, nearly $70 \%$ felt that their health was worse than it was one year ago. Finally, the vast majority of the aged in the Wuhan area were at least somewhat satisfied with their health, and only $22 \%$ were not very or rot at all satisfied.

Consistent with the statistics pertaining to IADL and physical fitness, the young old, male, those married, and urban residents considered themselves in better physical health in general and when compared with peers their age and their prior health status one year before, and they were more contented with their own health.

\section{Evaluation of Structural Model}

Although descriptive analyses are informative, structural equation modeling is essential in assessing (1) the multidimensional nature of physical health, (2) measurement errors associated with self-reported physical health, and (3) direct and indirect effects of socioeconomic variables on physical health. More importantly, structural equation analysis provides an empirical basis for evaluating the goodness-of-fit of the proposed model to the data obtained in Wuhan, China. See Bollen (1989) for a detailed discussion of structural equation modeling and its interpretation.

Table III contains the parameter estimates and goodness-of-fit indices for the proposed model (Figure 1). In general, the model $\left(\mathbf{M}_{1}\right)$ is supported by the results. Several measures of fit were used to assess the goodness-of-fit of the proposed model. With 18 degrees of freedom, the probabilities associated with the likelihood ratio are zero in both subsamples, indicating that $M_{1}$ does not fit the data well (Table III). Given that the likelihood ratio is very sensitive to sample size, measures of fit adjusted for the sample size are more appropriate. These measures include the Goodness-of-Fit Index (GFI) and Adjusted Goodness-of-Fit Index (AGFI) developed by Jöreskog and Sörbom (1988), and the normed and unnormed fit indices $\left(\Delta_{1}\right.$ and $\left.\Delta_{2}\right)$ devised by Bentler and 
TABLE III

Unstandardized and standardized maximum likelihood estimates for factor loadings and measures of fit for the various models $\left(\mathrm{M}_{1}\right)$

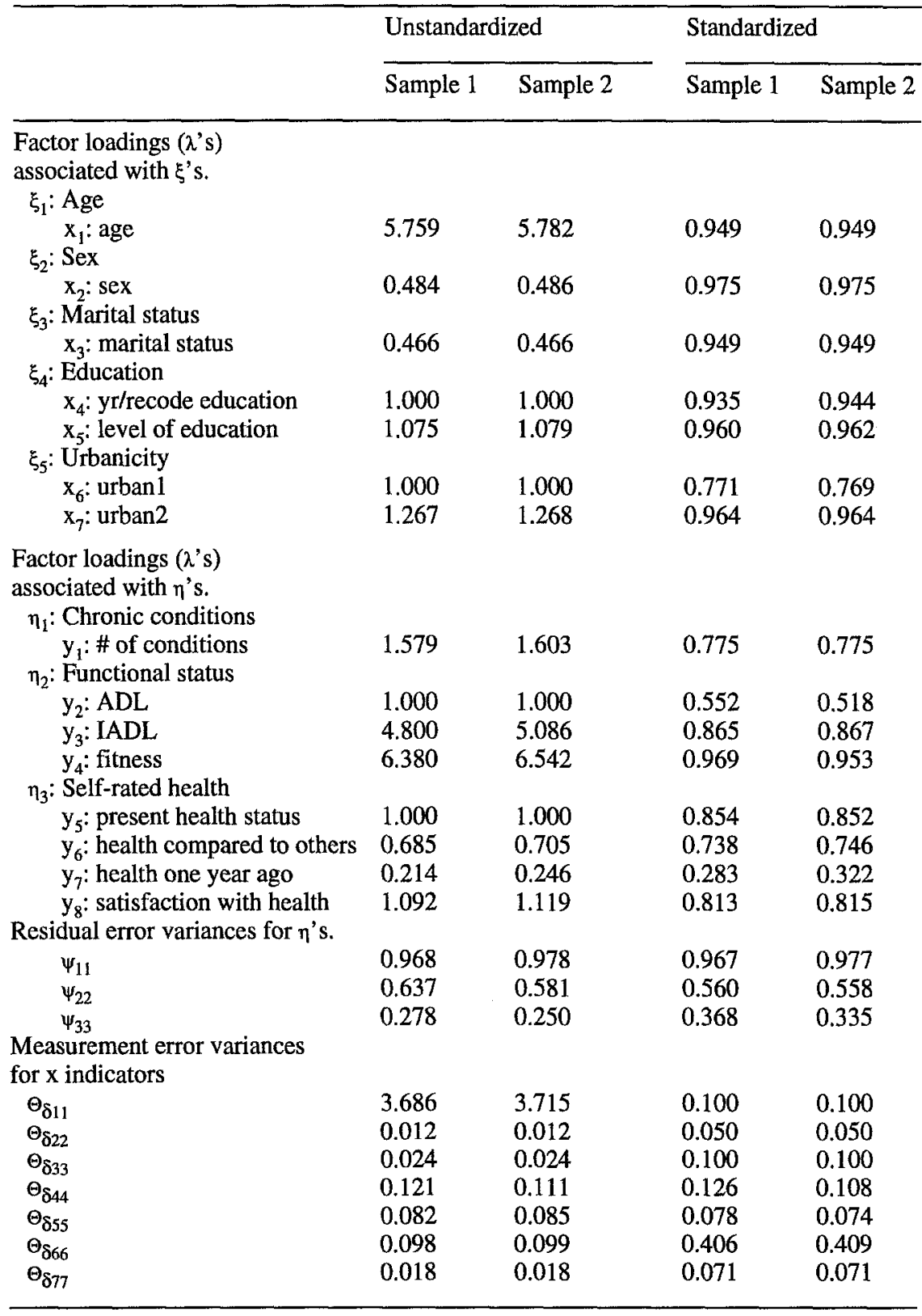




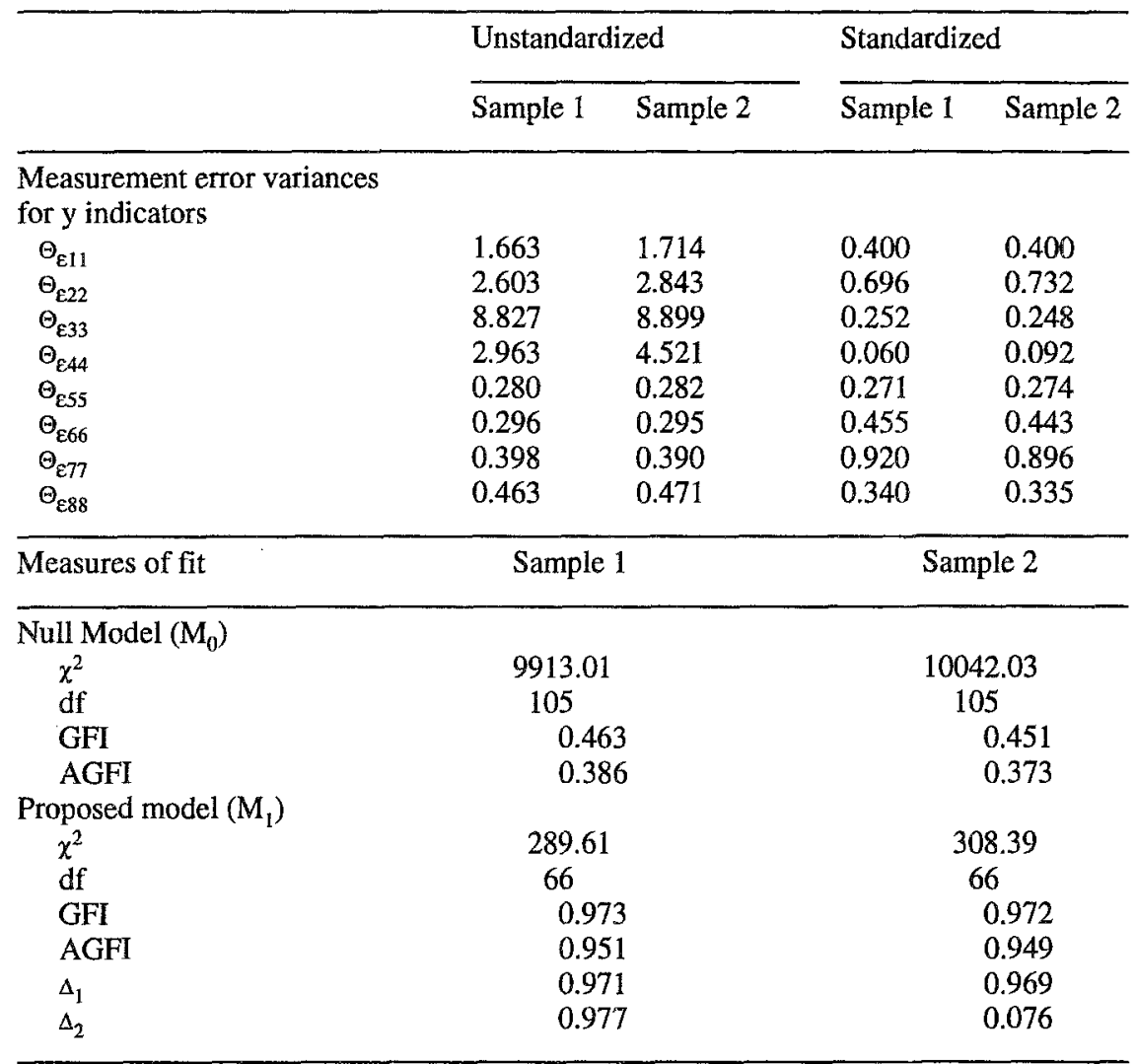

Note: Estimates associated with factor loadings are significant at $\mathrm{p}<0.001$. To conserve space, item wordings are compressed.

$\chi^{2}=$ Chi-square; df $=$ degrees of freedom; GFI = Goodness-of-Fit Index; AGFI = Adjusted Goodness-of-Fit Index; $\Delta_{1}=$ Bentler-Bonett Normed Index; $\Delta_{2}=$ Bollen NonNormed Index

Bonnett (1980), and Bollen (1989). As shown in Table III, the values of GFI, AGFI, $\Delta_{1}$, and $\Delta_{2}$ for $M_{1}$ are equal to or greater than 0.95 , indicating an excellent fit.

In addition to the overall fit measures, attention needs to be directed to the component fit measures. Generally the parameter estimates are consitent with the empirical predictions derived from the proposed measurement model $\left(M_{1}\right)$. Table III presents the standardized maximum likelihood estimates of parameters for both samples. All estimates of factor loadings are at least statistically significant at the 0.05 level, and are in the expected direction. With the exception of the loading of $y_{7}$ (i.e., "health a year ago"), all loadings are greater than 0.518 . The loadings associated with $y_{7}$ range from 0.283 to 0.322 . With reference to the causal linkages among the three latent health dimensions, all coefficients are also statistically significant at the 0.05 level and in the expected 
JERSEY LIANG ET AL.

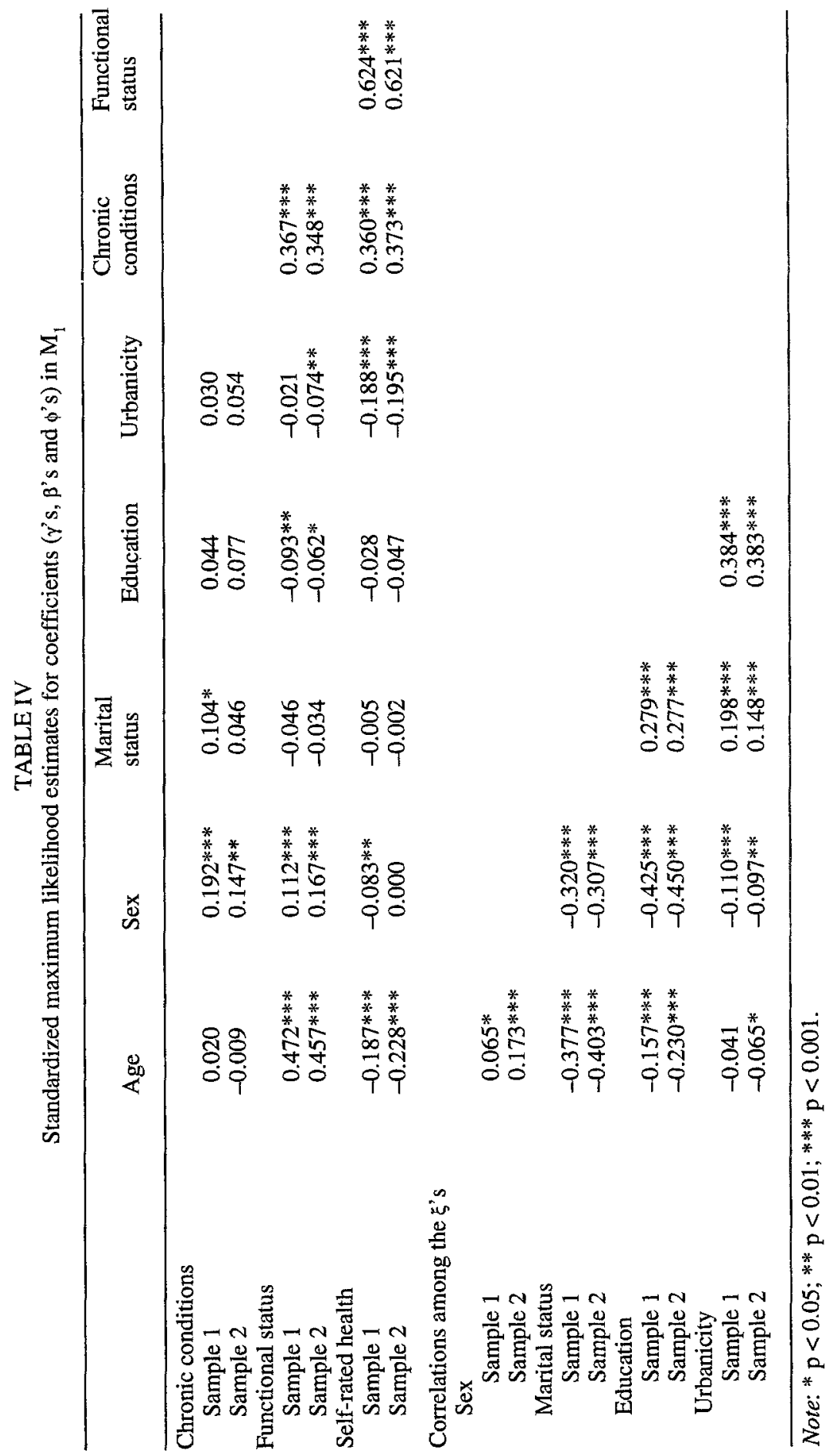


direction (see Table IV). Whereas approximately $44 \%$ of the variance in functional status is accounted for in both subsamples, 63 to $67 \%$ of the variance in self-rated health (subsample 1 and 2, respectively) is explained in the proposed model $\left(\mathrm{M}_{1}\right)$. These findings are consistent with previous research on the U.S. and Japanese elderly (Liang et al. 1991).

Standardized maximum likelihood estimates for the effects of the socioeconomic characteristics on the health dimensions are presented in Table IV. Gender is the only socioeconomic factor which has a consistent and statistically significant effect on chronic disease. Specifically, Chinese females have significantly higher levels of chronic conditions than do males. In addition, being older, female, and having a greater number of chronic conditions is related to greater functional limitations. A modest effect of education is also revealed: less educated Chinese suffer greater functional limitations. Results regarding the impact of urbanicity on function are inconclusive. Being older is significantly related to better self-rated health, after controlling for chronic conditions and functional status. Finally, living in a rural setting, having a greater number of conditions and greater functional limitations results in poorer self-rated health among the Chinese elderly. In summary, the findings suggest that the older old, women, rural residents, and those with little education have worse health.

\section{DISCUSSION}

This study contributes to research on physical health status in the Chinese elderly specifically and physical health status in the elderly in general in several respects. First, a comprehensive enumeration of chronic diseases, functional status, and self-rated health in a representative sample of aged people in the Wuhan area, China was presented. Although there are substantial differences in self-reported diseases across age, sex, marital status, and residence groups, the older old, women, those not married, and rural dwellers appear to have worse health in terms of disability and self-rated health.

Second, a three-dimensional structure of physical health previously evaluated in the United States and Japan (Liang et al. 1991) was replicated with data collected in the aged in the Wuhan area, China. Although identical measures are not used in all three countries, the results for China are very similar to those in the U.S. and Japan in terms of the measurement specifications (Liang et al. 1991). Accordingly, this three-dimensional physical health model has been shown to be quite robust and can be generalized to the Chinese elderly in addition to the elderly Americans and Japanese. This similarity in the structure of physical health certainly facilitates a more meaningful comparison across these three different cultures.

Third, results of structural equation analyses suggest that variables such as age, sex, and education influence chronic diseases, disability, and self-rated health directly and/or indirectly. For instance, age affects self-rated health directly and indirectly through its effect on functional status (Table IV). Whereas the direct effect of sex on self-rated health is somewhat inconclusive, 
sex influences self-rated health indirectly through its impact on chronic diseases and disability (Table IV). Education has a direct impact on functional status and an indirect effect on self-rated health via functional status.

Fourth, our findings regarding sex differences in self-rated health are consistent with prior findings derived from the U.S. On the basis of bivariate crosstabulations, women tend to rate their health worse than men. Once sociodemographic and other health characteristics are controlled, sex differences in self-rated health tend to diminish considerably. It is not uncommon that such sex differences become non-significant or even reversed (Verbrugge 1989). According to our descriptive analyses in Table II, the old old and women did rate their health to be worse than the young old and men. In our structural analysis where chronic diseases, functional status, and a host of sociodemographic variables are controlled, sex differences are no longer statistically significant.

How do the elderly Chinese respondents fare in self-reported physical health status when compared with their peers in the United States and Japan? Somewhat parallel analyses of physical health status using data from national samples of older people in the U.S. and Japan were undertaken previously (Liang et al. 1991). Although not all items nor all analyses were identical, comparisons of the above findings with those from the U.S. and Japan are nevertheless useful in further explicating their meaning.

Although many differences in the pattern of prevalence among the three countries existed, there were several of note. First, for the conditions measured in China and Japan, all diseases except high blood pressure are represented by a higher percentage of Chinese elderly than was the case for the Japanese elderly. Results for the U.S. were mixed. In addition, the Chinese aged experience higher levels of co-morbidity than the elderly in the U.S. and Japan (Liang et al. 1991).

Second, like their counterparts in the U.S. and Japan, the Chinese elderly evidence only a small amount of ADL limitations, however, they reported more difficulty with IADL than the aged in Japan and the U.S. Further, in all cases the Japanese had a higher percentage of elderly able to perform the fitness activities with no difficulty when compared to both China and the U.S., but the Chinese fared better when compared to the U.S. elderly. Finally, although a greater percentage of the Chinese aged felt their health had deteriorated more than their U.S. counterparts, it appears as if they nevertheless rate their present health as better than the elderly in the U.S. Percentages in China, however, were much more similar to those in Japan than was the case for the U.S., although some differences were still evident (Liang et al. 1991).

What are the similarities and differences in the effects of socioeconomic factors on health status between the aged in the Wuhan area and elderly in the U.S. and Japan? On the basis of the present findings and recent results reported by Liang and his associates (1991), the following comparisons and contrasts can be made. As was the case with their age peers in the U.S. and Japan, the older old in the Wuhan area rated their health to be better. On the other hand, unlike the findings from the U.S. and Japan, there was no age difference in terms of 
chronic conditions among the aged in the Wuhan. In addition, the age effect on functional status was significant in the aged in the Wuhan area and Japan but not significant in the aged Americans.

In all three countries, no statistically significant sex difference in self-rated health was found. However, sex differences in chronic conditions and disability were more apparent in the Wuhan area than in the U.S. and Japan. In the Wuhan area, elderly women were found to suffer from more chronic diseases and disability. With the exception of a sex difference in the prevalence of chronic disease observed in the U.S., no such differences were found in the American and Japanese aged (Liang et al. 1991).

Marital status has consistently been shown to have no direct effect on all three dimensions of physical health in the elderly in the U.S., Japan, and the Wuhan area. In addition, no educational difference in the prevalence of chronic diseases was observed in any of the three countries. However, in the Wuhan area and the U.S., those with more education tended to have less disability, whereas an educational effect on self-rated health was found only in the elderly Americans.

In view of the above contrasts, there appear to be cross-national differences (1) in the effects of age and sex on the prevalence of chronic disease and disability and (2) in the effects of education on disability and self-rated health. Due to the lack of theories and empirical research, substantive and methodological accounts for these observed cross-cultural differences are difficult to provide. Instead, we suggest some general directions of and hypotheses for further research. It is our firm belief that the present research represents a necessary initial step toward the formulation of more specific hypotheses and analytical models that hopefully will provide additional insights concerning the underlying causal mechanisms accounting for the observed differences.

At least two types of explanations need to be entertained, epidemiological and sociocultural. Epidemiologically, our findings reveal the differential roles of age, sex, and education as risk factors for physical health in the Wuhan area, the U.S., and Japan. The effects of these factors may reflect cross-cultural differences in genetics, nutrition, health behavior, health care, and environment. Socioculturally, one may examine differences in the definition of and attitude toward illness and health between the Chinese, American, and Japanese elderly. More importantly, attention needs to be directed to the connections between individual characteristics and sociocultural variations in pertinent dimensions including values, social structure, technology, and population.

On the other hand, our observations need to be further replicated before they can be considered as genuine cross-cultural differences. This cautious approach can be justified on the following grounds. Although there is a strong indication that the structure of physical health is conceptually similar in the Wuhan area, the United States, and Japan, its metric or measurement equivalence has not been substantiated due to the differences in the health indicators employed in these three different cultural settings. Accordingly, the observed differences in relationships between physical health and selected exogenous variables may be confounded by possible measurement non-equivalence. 
It should be noted that the data on physical health in the Chinese aged were collected largely within a framework developed by the American investigators. Although the validity of this framework is supported by our analyses, additional research can be undertaken to further evaluate the meaningfulness of this scheme within the Chinese culture. In particular, efforts should be made to explore new dimensions as well as indicators of health status unique to the Chinese society. Conceivably, the construct of health status can be defined and operationalized in China without making any reference to measures developed elsewhere. In this respect, the triangulation of both quantitative and qualitative approaches can be particularly fruitful. Such analyses may also yield additional insights concerning the cross-cultural comparability in the definitions and measures of physical health.

As the present research involved a probability sample of only the aged in the Wuhan area, China, the extent to which our findings can be generalized to the total elderly population in China needs to be addressed. The differences between the Wuhan area sample and the total aged population in China can be assessed by contrasting their socioeconomic characteristics. Whereas the Wuhan area sample is similar to the Chinese aged in general in terms of age structure and marital status, it differs from the total aged population in several ways (Population Research Institute, CASS 1988; State Statistical Bureau of the People's Republic of China 1991). First, the Wuhan area sample contains a substantially higher population proportion of urban elderly (59\%) than the nation as a whole $(26 \%)$. As a consquence, the respondents in the Wuhan area sample are likely to be better educated. Second, the sex ratio associated with the Wuhan area sample (84\%) is lower than that for the total elderly Chinese population $(89 \%)$. Although such differences may render the frequency distributions derived from the Wuhan area sample less representative of the Chinese aged as a whole, this is less of a problem with the results of multivariate analyses because population heterogeneity has been controlled. However, this does not elimiante the need to replicate the survey in other sites in China or to undertake a national probability sample survey of the Chinese aged.

On the other hand, one must recognize that presently there is no high quality national or even regional data on physical health in the Chinese aged. In contrast to previous studies of the Chinese aged, the present research is unique in that it was based upon a large scale probability sample survey involving both the urban and rural elderly. The present research is a necessary step toward the establishment of a high quality national data base of the Chinese aged.

Finally, a sensible analysis of health status in the Chinese aged requires a keen awareness of the unique social, political, and demographic characteristics which include a centralized policy-making system, a large rural population, and a fast growing private sector. In addition, population aging in China coincides with other processes such as increased industrialization, urbanization, political reform, and epidemiological transformation. During the past decade, China has undergone major changes in areas of economic development, community organization, bureaucracy, and social stratification (Walder 1989). The inter- 
relationship among these societal trends, old age support, and the well-being of the aged in China are critical research and policy issues yet to be addressed.

\section{ACKNOWLEDGEMENT}

This research was supported by grants R01 AG08094 and R37 AG06643 provided by the U.S. National Institute on Aging. The assistance provided by Dennis Chuang and Cathy Fegan is gratefully acknowledged.

\section{REFERENCES}

Angel, R., and P. Thoits 1987 The Impact of Culture on the Cognitive Structure of Illness. Culture, Medicine and Psychiatry 11: 465-494.

Bentler, P.M., and D.G. Bonnett 1980 Significance Tests and Goodness-of-Fit in the Analysis of Covariance Structures. Psychological Bulletin 88: 588-606.

Bollen, K.A. 1989 Structural Equations With Latent Variables. New York: Wiley.

Banister, J. 1988 Implications of the Aging of China's Population. Center for International Research Staff Paper No. 44, U.S. Bureau of Census, Washington, D.C.

Coale, A.J. 1984 Rapid Population Change in China, 1952-1982. Washington, D.C.: National Academy Press.

Ferraro, K.F. 1988 Self-ratings of Health Among the Old and Old-old. Journal of Health and Social Behavior 42: 52 .

Fillenbaum, G. 1979 Social Context and Self Assessment of Health Among the Elderly. Journal of Health and Social Behavior 20: 45.

Grigsby, J.S., and S.J. Olshansky 1989 The Demographic Components of Population Aging in China. Journal of Cross-Cultural Gerontology 4: 307-334.

Gu, S., and J. Guo 1989 A Comprehensive Study of the Urban Elderly. Wuhan, China: Wuhan University Press.

Gu, S., and B. Wang 1989 International Comparison of the Health and Social Life of the Elderly. Wuhan, China: Wuhan University Press.

Gui, S.X., L.K. Ki, L.N. Shen, J.X. Di, Q.Z. Gu, Y.M. Chen, and F. Qian 1987 Status and Needs of the Elderly in Urban Shanghai: Analysis of Some Preliminary Statistics. Journal of Cross-Cultural Gerontology 2: 171-186.

Ikels, C. 1991 Aging and Disability in China: Cultural Issues in Measurement and Interpretation. Social Sciences and Medicine 32: 649-665.

International Assistance Group on Family Planning 1989 Chartbook of Population Aging in Asia. Tokyo, Japan.

Jaing, Z., W. Zhang, and L. Zhu 1984 A Preliminary Examination of Life Expectancy in China. Ren Kou Yu Jing Ji (Population and Economics) 3: 14-17.

Jamison, D.T., J.R. Evans, T. King, I. Porter, I. Prescott, and A. Prost 1984 China: The Health Sector. Washington, D.C.: World Bank.

Jöreskog, K.G. and D. Sörbom 1988 LISREL 7: A Guide to the Program and Applications. Chicago, IL: SPSS Inc.

Kantha, S. 1990 Nutrition and Health in China, 1949 to 1989. Progress in Food and Nutrition Science 14: 93-137.

Kohn, M.L. 1987 Cross-National Research as an Analytic Strategy. American Sociological Review 52: 713-731.

Liang, J. 1986 Self-Reported Physical Health Among the Aged Adults. Journal of Gerontology 41: 248-260. 
Liang, J., J. Bennett, N. Whitelaw, and D. Maeda 1991 The Structure of Self-Reported Physical Health Among the Aged in the United States and Japan. Medical Care 29: 1161-1179.

Liang, J., S. Gu, and N. Krause 1992 Social Support Among the Aged in Wuhan, China. Asia-Pacific Population Journal 7: 33-62.

Liang, J., J.C. Tu, and X. Chen 1986 Population Aging in the People's Republic of China. Social Science and Medicine 23: 1353-1362.

Lock, M.M. 1987 Introduction: Health and Medical Care as Cultural and Social Phenomena. In Health, Illness, and Medical Care in Japan. Edward Norebeck and Margaret Lock, eds. Pp.1-23). Honolulu: University of Hawaii Press.

Markides K., and H. Martin 1979 Predicting Self-Rated Health Among the Aged. Research on Aging 1: 97.

Pope, A.M., and A.R. Tarlov 1991 Disability in America: Toward a National Agenda for Prevention. Washington, D.C.: National Academy Press.

Population Research Institute, Chinese Academy of Social Sciences 1988 The 1987 Sample Survey of Population 60 Years and Over in China. Population Science of China. Special Issue No. 1.

Qu, H. 1984 Status of the Elderly in China. Cairo Demographic Center Research Monography Series, No. 13, Cairo.

State Statistical Bureau of the People's Republic of China 1991 China Statistical Yearbook, 1991. Beijing, China: International Center for the Advancement of Sciences and Technology Ltd. and China Statistical Information and Consultancy Services Center.

Stoller, E.P. 1984 Self-assessments of Health by the Elderly: The Impact of Informal Assistance. Journal of Health and Social Behavior 25: 260.

U.S. Bureau of Census 19751970 Census of Population and Housing. Evaluation and Research Program, Vol. 11: Accuracy of Data for Selected Population Characteristics as Measured by the 1970 CPS-Census Match. PHC(E)-11. Washington, DC: Government Printing Office.

U.S. Senate Special Committee on Aging 1988 Aging America: Trends and Projections. Washington, D.C.: U.S. Department of Health and Human Services.

Verbrugge, L.M. 1989 The twin meet: Empirical Explanations for Sex Differences in Health and Mortality. Journal of Health and Social Behavior 30: 282-304.

Walder, A.G. 1989 Social Change in Post-Revolution China. Annual Review of Sociology 15: 405-424.

Whitelaw, N.A., and J. Liang 1991 The Structure of the OARS Physical Health Measures. Medical Care: 659-676.

World Health Organization 1958 The First Ten Years of World Health Organization. Geneva: World Health Organization.

$\mathrm{Xu}, \mathrm{S} .1985$ Health Statistics of the People's Republic of China. In Good Health at Low Cost. S.B. Halstead, J.A. Walsh and K.S. Warren, eds. Pp. 11-20. N.Y.: Rockefeller Foundation.

Yang, H. 1989 The Family Support System for the Elderly in Rural China. Unpublished doctoral dissertation.

Yang, P.L., V. Lin, and J. Lawson 1991 Health Policy Reform in the People's Republic of China. International Journal of Health Service 21(3): 481-491.

Jersey Liang and Joan Bennett

Institute of Gerontology

and School of Public Health

The University of Michigan

Ann Arbor, Michigan 48109-2007 
Shengzu Gu

Institute for Population Research

and

Center for Social and Economic Research

Wuhan University

Hubei, Wuhan

People's Republic of China 